\title{
Themenspektrum von HeilberufeSCIENCE
}

\author{
Jörg Klewer
}

Online publiziert: 20. Januar 2015

(C) Springer-Verlag Wien 2015

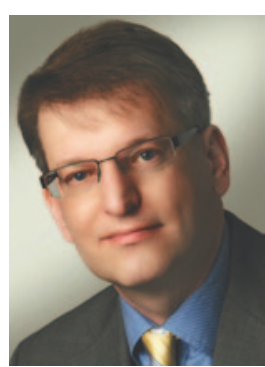

Jörg Klewer

Mit dieser Ausgabe beginnt der 6. Jahrgang von HeilberufeSCIENCE. Auch im Jahr 2015 erwarten Sie 4 reguläre Ausgaben und ein Supplement mit den Abstracts des 5. HeilberufeSCIENCE-Symposiums. Unser Ziel ist es weiterhin, Ihnen als Leser Publikationen von hoher inhaltlicher Qualität zu interessanten wissenschaftlichen Ergebnissen aus den Gebieten Pflegemanagement, Pflegepädagogik, Pflegewissenschaften, Gerontologie, Altenpflege und Gesundheitswissenschaften/Public Health zu bieten.

Sicherlich stellt sich immer wieder die Frage, ob fachliche Breite auch mit inhaltlicher Tiefe einhergehen kann, oder ob nicht sinnvollerweise eine thematische Eingrenzung erfolgen sollte. $\mathrm{Zu}$ beiden Positionen gibt es Pro- und Kontraargumente, jedoch bestand eine der Intentionen von HeilberufeSCIENCE darin, insbesondere dem wissenschaftlichen Nachwuchs eine Plattform zu bieten. Da oftmals zu Beginn der persönlichen akademischen Karriere eine eigene thematische Einordnung schwierig ist, soll deshalb die breit gefächerte Ausrichtung von Heilbe-

J. Klewer $(\bowtie)$

Zwickau, Deutschland

E-Mail: joklewer@t-online.de
rufeSCIENCE den Einstieg in die „scientific community“ erleichtern.

In der vorliegenden Ausgabe von HeilberufeSCIENCE bieten wir Ihnen daher 4 Publikationen und eine wissenschaftliche Kurzmitteilung aus dem Themenspektrum von HeilberufeSCIENCE. Die englischsprachige Originalarbeit von Schaal et al. widmet sich am Beispiel von deutschen Pflegeheimen der Erfassung von pflegerischer Versorgung; hierbei handelt es sich um ein Thema von mittlerweile hoher internationaler Relevanz. Die Übersichtsarbeit von Brandstetter hat ebenfalls den älteren Menschen im Fokus und präsentiert die Ergebnisse einer Literaturrecherche $\mathrm{zu}$ Möglichkeiten der Erfassung des Schmerzverhaltens von Menschen mit schwerer Demenz. Hierbei zeigt er, dass zum derzeitigen Zeitpunkt noch keine umfassend geeigneten Instrumente existieren, sodass weiterer Forschungsbedarf besteht.

Auf die Gruppe der Auszubildenden in der Krankenpflege gehen Lehmann et al. in ihrer Originalarbeit ein, indem sie Verbindungen zwischen dem Gesundheitsverhalten der Auszubildenden und deren sozioökonomischem Status untersucht haben. Eine entsprechende Assoziation fand sich jedoch nur für den Zigarettenkonsum. Nach Ansicht der Autoren müsste eingehender untersucht werden, inwieweit die Auswahl von Auszubildenden zu einer Unterrepräsentation von Auszubildenden mit geringem sozioökonomischen Status geführt haben kann. In einer systematischen Übersichtsarbeit gehen Posch-Eliskases et al. auf Stressoren für pflegende Angehörige ein und leiten daraus geeignete Maßnahmen ab, wie pflegende Angehörige idealerweise auf die pflegerische Tätigkeit vorbereitet werden sollten. Eine methodisch ausgerichtete wissenschaftliche Kurzmitteilung von Meyer et al. untersuchte beispielhaft in einem Krankenhaus das Thema der Aufnahme der Essensbestellung durch Servicemitarbeiter. 
Obwohl die Verlagerung von pflegerischen Tätigkeiten auf Servicepersonal in vielen Krankenhäusern zunehmend $\mathrm{zu}$ beobachten ist, wurde dieser Bereich bislang kaum beleuchtet.

Hiermit lade ich Sie wieder ein, die 5 wissenschaftlichen Arbeiten dieser Ausgabe von HeilberufeSCIENCE kritisch $\mathrm{zu}$ lesen und in den wissenschaftlichen Dialog einzutreten.
Zudem würde es mich freuen, Sie im April zum 5. HeilberufeSCIENCE-Symposium auf dem Interprofessionellen Gesundheitskongress in Dresden begrüßen zu dürfen.

Ihr

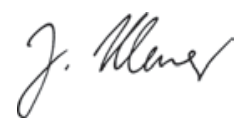

